\title{
STOCK DE CARBONO EN UN PASTIZAL DE Sorghastrum setosum (Griseb.) Hitchc EN LA PROVINCIA DE CHACO, ARGENTINA.
}

\author{
Céspedes Flores, Flora ${ }^{1}$; Bernardis, Aldo ${ }^{1}$; Fernández, Juan¹; Gobbi, José2; Roig, Carlos² \\ ${ }^{1}$ Facultad de Ciencias Agrarias - UNNE. fcespedes@agr.unne.edu.ar \\ ${ }^{2}$ EEA INTA Colonia Benítez (Chaco)
}

\section{RESUMEN}

El objetivo de este trabajo fue evaluar el contenido de carbono en suelo y biomasa aérea de un pastizal de Sorghastrum setosum. El ensayo se realizó en el Campo Anexo General Obligado, dependiente de la E.E.A. INTA Colonia Benítez, ubicado en el Departamento General Dónovan (Chaco). El área corresponde al Parque Chaqueño Húmedo, posee un clima templado húmedo sin estación seca. El suelo pertenece a la Serie Charadai, es un natracualf típico. La vegetación predominante es un pastizal de Sorghastrum setosum. El diseño estadístico fue un DBCA con 5 repeticiones. Las variables medidas fueron contenido de Carbono y Densidad Aparente (DA) del suelo y contenido de Carbono en la biomasa aérea del pastizal. Los resultados de contenido de Carbono en suelo fueron: a $0,1 \mathrm{~m}$ de profundidad de $24,35 \mathrm{tn} \mathrm{ha}^{-1}$, y a $1 \mathrm{~m}$ de 40,25 tn ha ${ }^{-1}$; la DA en estos estratos fue de $1,12 \mathrm{tn}^{3}$ a $0,1 \mathrm{~m}$ y $1,29 \mathrm{tn}^{3}$ a $1 \mathrm{~m}$ de profundidad; el contenido de Carbono en la biomasa aérea del pastizal fue de 5,65 tn ha ${ }^{-1}$. El stock de Carbono en primavera en el ecosistema del pastizal es 139,24 tn ha-1, donde 95,94\% corresponde a Carbono acumulado en suelo y el resto en menor proporción a Carbono acumulado en la biomasa aérea del pastizal.

PALABRAS CLAVE: pastoreo, Carbono en el suelo, humus, cambio climático.

\begin{abstract}
The aim of this study was to evaluate the content of soil Carbon and aerial biomass of a Sorghastrum setosum pasture. The study took place at Campo Anexo "General Obligado", E.E.A. INTA Colonia Benitez, located in Departamento General Dónovan (Chaco). The area belongs to The Humid Chaqueño Park, the climate is humid temperate without dry season. The soil type corresponds to the Charadai Series, being a typical Natracualf. The predominant vegetation is grassland of Sorghastrum setosum. The statistical design was a DBCA with 5 replications. The measured variables were soil Carbon and Soil Apparent Density (DA) and Carbon in aerial biomass. The results in soil Carbon were: up to $0,1 \mathrm{~m}$ depth, of $24.35 \mathrm{tn} \mathrm{ha}^{-1}$; and up to $1 \mathrm{~m}$, of $40,25 \mathrm{tn} \mathrm{ha}^{-1}$. The DA
\end{abstract}

in these strata was: $1.12 \mathrm{tn} / \mathrm{m}^{3}$ at $0,1 \mathrm{~m}$ depth, and $1.29 \mathrm{tn} / \mathrm{m}^{3}$ at $1 \mathrm{~m}$ depth; the Carbon in aerial biomass was of $5.65 \mathrm{tn} \mathrm{ha}^{-1}$. The content of Carbon in spring in Sorghastrum setosum grassland is 139.24 tn ha $^{-1}$, where $95.94 \%$ correspond to accumulated soil Carbon and the rest in smaller proportion to accumulated Carbon in the aerial biomass of the grassland.

KEY WORDS: grazing, soil Carbon, humus, climatic change.

\section{INTRODUCCIÓN}

Los pastizales contribuyen a mitigar el cambio climático global almacenando Carbono en la biomasa y en el suelo por el proceso de la fotosíntesis y el ciclo del Carbono (Conant et al., 2005).

Los llamados gases de efecto invernadero tienen la capacidad de atrapar la radiación solar dentro de la tierra y su aumento puede causar un incremento de temperaturas medias a nivel global y producir cambios en la vegetación y zonas ecológicas. Se prevé que a mediados del siglo XXI el incremento del dióxido de Carbono $\left(\mathrm{CO}_{2}\right)$ duplicará al de la era preindustrial, lo que producirá un aumento de temperaturas de 1,5 a $4,5^{\circ} \mathrm{C}$ (IPCC, 2001a, b, c). Entre los gases invernaderos naturales el principal es el $\mathrm{CO}_{2}$, cuyo nivel se ve incrementado debido a actividades humanas como el consumo de combustibles fósiles, cambios en el uso de la tierra, la deforestación y la agricultura, entre otros (Houghton et al., 2001).

La materia orgánica del suelo (MOS) es un indicador clave de la calidad del suelo, tanto en sus funciones agrícolas (ej. producción) como en sus funciones ambientales - entre ellas captura de Carbono y calidad del aire. La materia orgánica disminuye en los suelos cultivados, principalmente en la capa de suelo labrada, debido a una caída de los aportes de residuos, al incremento de la temperatura y a la disrupción de agregados (Dixon, 1995). Actualmente los estudios en la MOS apuntan a establecer la capacidad de la misma de almacenar Carbono del aire y contribuir a mitigar el calentamiento global (FAO, 2002).

En los pastizales, una de las medidas que puede incrementar en buena medida la captura de Carbono es la introducción de especies forrajeras más produc- 
tivas, con sistemas radicales más profundos, y altamente persistentes al pastoreo, ya que éstas pueden almacenar muy altas cantidades de Carbono en forma estable (Fisher et al., 1994).

La región del noreste argentino (NEA), está integrada por las provincias de Formosa, Chaco, Corrientes y Misiones. El pastizal natural es la base forrajera de la ganadería de esta región, la calidad y disponibilidad, definen el tipo de ganadería que prevalece en la misma. Una característica es que están compuestos casi exclusivamente por especies estivales, como los pajonales de Sorghastrum setosum (Griseb.) Hitchc (Bernardis et al., 2005). No existe información sobre el stock de Carbono en los pastizales de la región, ni tampoco sobre la capacidad de mitigar el efecto invernadero que tienen los pastizales. Este trabajo se presenta como una línea base respecto a este tema, evaluando la condición actual en la que se encuentran los pastizales en la provincia del Chaco.

El objetivo de este estudio fue evaluar el contenido de Carbono en suelo y en la biomasa aérea de un pastizal de paja amarilla.

\section{MATERIALES Y MÉTODOS}

El ensayo se realizó en el Campo Anexo General Obligado, dependiente de la E.E.A. INTA Colonia Benítez, ubicado en el Departamento General Dónovan, provincia del Chaco, Argentina. El campo se encuentra en el parque Chaqueño oriental (Chaco húmedo), con precipitación media de $1.300 \mathrm{~mm}$ anuales y temperatura media de $21,5^{\circ} \mathrm{C}$. El período libre de heladas es de 354 días; posee un clima templado húmedo sin estación seca (Cabrera, 1994). El suelo corresponde a la Serie Charadai, es un Natracualf típico. Tiene un horizonte superficial lixiviado, color gris claro, textura pesada y media; un subsuelo gris, textura pesada, con abundantes concreciones y muñequillas de carbonato de calcio (Ledesma y Zurita, 1995). La vegetación predominante es un pastizal de S. setosum.

Los muestreos se realizaron en primavera, en parcelas de clausura de $25 \mathrm{~m}^{2}$ cada una (Figura 1 a). Se realizaron muestreos de biomasa aérea en las 5 parcelas (bloques) dentro del campo, para evaluar el contenido de Carbono en esa época del año. Las muestras de biomasa aérea se obtuvieron por medio de un cuadro de $1 \mathrm{~m}^{2}$ tirado al azar dentro de cada bloque, realizando los cortes con tijera al ras del suelo. Se tomaron muestras de suelo en cada parcela cavando calicatas hasta $1 \mathrm{~m}$ de profundidad, definiendo cuatro tosum. profundidades de muestreo: $0-0,10 \mathrm{~m}, 0,10-0,20 \mathrm{~m}$, $0,20-0,60 \mathrm{~m}$ y $0,60-1 \mathrm{~m}$ (Figura 1 b). Se determinó la Densidad Aparente de las muestras de suelo por medio de cilindros de Kopecky (Prause, 2006). Se obtuvieron 20 (veinte) muestras de suelo, de las cinco parcelas muestreadas a cuatro profundidades en cada una, y cinco muestras de biomasa aérea del pastizal en el área de muestreo.

El material verde cortado de la biomasa aérea, se pesó y se llevó a estufa a $65^{\circ} \mathrm{C}$ hasta peso constante, para determinar la Materia Seca (MS). El contenido de Carbono en la biomasa aérea del pastizal se calculó de la siguiente manera (Álvarez, 2006):

$$
\mathrm{C}=\mathrm{B} \times 0,45
$$

\section{Donde}

$\mathrm{C}=$ Contenido de Carbono

$\mathrm{B}=$ Biomasa vegetal aérea (tn MS. ha ${ }^{-1}$ )

$0,45=$ Constante. Proporción de Carbono, asumido por convención del IPCC (2000) en la biomasa de pastizales.

El material orgánico de las muestras de suelo se separó por medio de tamices (Cambardella y Elliot, 1992), y el contenido de Carbono en las muestras se determinó por el método de Walkley-Black (1934). El contenido de Carbono (C) del suelo se estimó según Da Silva et al. (2004)

$$
\mathrm{C}=\mathrm{Conc} \times \mathrm{BD} \times \mathrm{T} \times 10^{-3} \times 10^{4}
$$

Donde

$\mathrm{C}=$ contenido de $\mathrm{C}\left(\mathrm{tn} \mathrm{ha}^{-1}\right)$

Conc $=$ contenido de Carbono en la muestra $\left(\mathrm{kg} \mathrm{tn}^{-1}\right)$

$\mathrm{BD}=$ densidad aparente de suelo $\left(\operatorname{tn~} \mathrm{m}^{-3}\right)$

$\mathrm{T}=$ profundidad de la muestra $(\mathrm{m})$
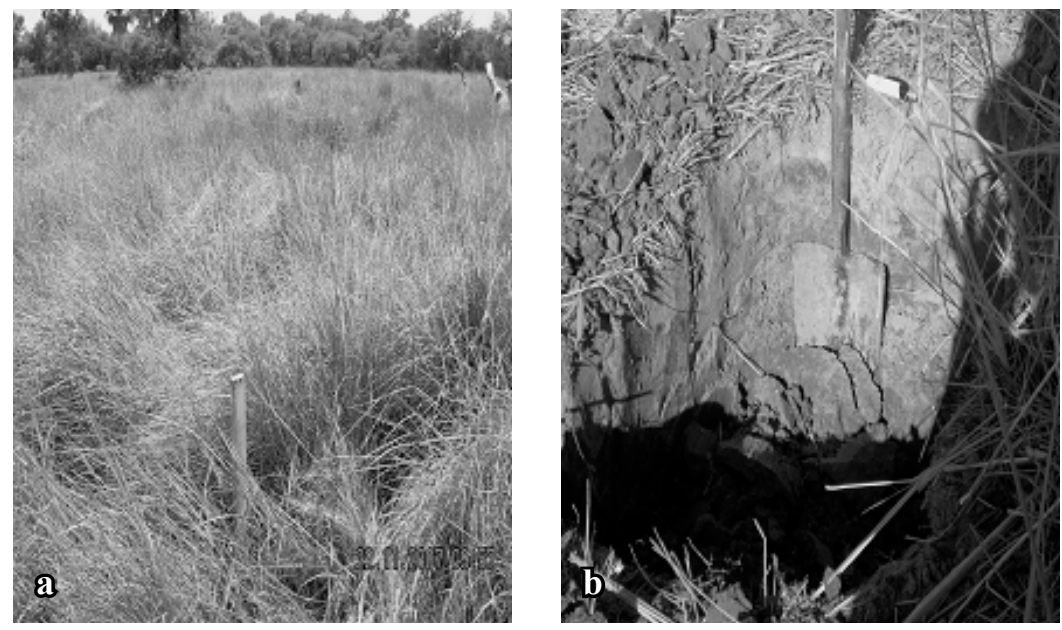

Figura 1: Lugar del sitio de muestreo. Foto a: pastizal de Sorghastrum setosum. Foto b: calicata de suelo bajo el pastizal de Sorghastrum se- 
Los resultados de biomasa aérea fueron descriptos a fin de evaluar el contenido de Carbono del pastizal en primavera. Con los datos obtenidos de suelo a distintas profundidades se realizó un ANOVA y comparación de medias a través de prueba de Tukey, utilizando InfoStat (2007).

\section{RESULTADOS Y DISCUSIÓN}

El contenido de Carbono total acumulado en suelo a $1 \mathrm{~m}$ de profundidad fue de 133,59 $\mathrm{tn} \mathrm{ha} \mathrm{h}^{-1}$ (Figura 2). Los valores de Carbono almacenado en el suelo difieren de aquellos hallados por Corazza et al. (1999) de 150 tn $\mathrm{ha}^{-1}$ y por Da Silva et al. (2004) de 97,1 tn ha ${ }^{-1}$, ambas evaluaciones hechas en pasturas cultivadas de Brachiaria decumbens sobre un Latosol Rojo Oscuro en la zona de El Cerrado, Brasil, los resultados de esta experiencia también difieren de las 200 tn ha ${ }^{-1}$ encontrados por Fisher et al. (1994), en las Sabanas Colombianas. Estas diferencias se deberían a los distintos usos de la tierra y al tipo de suelo estudiado en cada caso, y a la composición botánica que posee la Sabana. Corazza et al. (1999) y Da Silva et al. (2004) trabajaron con una pastura cultivada, Fisher $e t$ al. (1994) lo hicieron en un ecosistema natural donde existen pasturas cultivadas, nativas y consociaciones naturales con leguminosas, con una mayor riqueza de biomasa y por lo tanto mayor contenido de Carbono total en la sabana. Según Mannetje et al. (2008) las diferencias en cuanto a acumulación de Carbono en topografías de pendiente o planicies puede ser por un mal drenaje y acumulación de agua en llanos, o por impedimentos para la penetración de las raíces, por

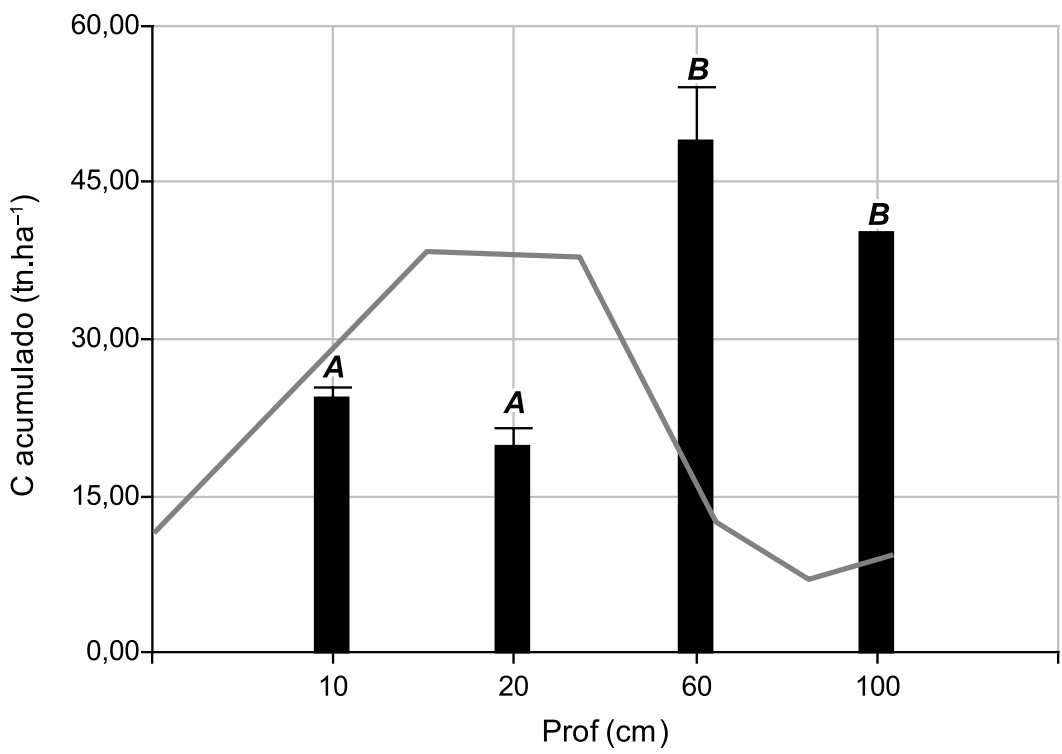

Figura 2: Carbono del suelo (C acumulado, tn $\mathrm{ha}^{-1}$ ) en un pastizal de Sorghastrum setosum (Barra), a diferentes profundidades de muestreo y contenido de Carbono de la serie de suelo Charadai (Línea). Letras distintas sobre las barras indican diferencias significativas entre las distintas profundidades $(\mathrm{p}<=0,05)$. lo cual tendría menos materia orgánica en profundidad y como consecuencia menor acumulación de Carbono. Las diferencias también podrían atribuirse a la topografía de suelo, ya que nuestro trabajo fue realizado en media loma y los estudios de Corazza et al. (1999), Da Silva et al. (2004) y Fisher et al. (1994) en llanuras.

Los resultados obtenidos de contenido de Carbono $\left(\mathrm{tn} \mathrm{ha}^{-1}\right)$ en las diferentes profundidades de muestreo fueron: $0-0,1 \mathrm{~m}=24,35 ; 0,1-0,2 \mathrm{~m}=19,87 ; 0,2$ $-0,6 \mathrm{~m}=49,12 ; 0,6-1 \mathrm{~m}=40,25$. El contenido de Carbono en suelo fue significativamente mayor en los primeros $0,6 \mathrm{~m}$ de suelo, el cual difirió de los valores obtenidos en las profundidades de $0,1 \mathrm{~m}$ y 0,2 $\mathrm{m}$. A partir de los $0,6 \mathrm{~m}$ hasta $1 \mathrm{~m}$ de profundidad el contenido de Carbono no varió significativamente. Más del $33 \%$ del Carbono acumulado en $1 \mathrm{~m}$ de profundidad se encuentra en los primeros $0,2 \mathrm{~m}$ de suelo (Figura 2).

La Densidad Aparente del suelo en estudio en las diferentes profundidades de muestreo fueron: $0-0,1$ $\mathrm{m}=1,12$ tn $\mathrm{m}^{3} ; 0,1-0,2 \mathrm{~m}=1,28$ tn $\mathrm{m}^{3} ; 0,2-0,6 \mathrm{~m}$ $=1,312 \mathrm{tn}^{3}$ y $0,6-1 \mathrm{~m}=1,29 \mathrm{tn} \mathrm{m}^{3}$.

Comparando los resultados obtenidos en esta evaluación con los datos de contenido de Carbono de la serie Charadai, referencia para estos ecosistemas, podemos decir que los resultados de Carbono encontrados en esta experiencia son mayores a aquellos publicados en la carta de suelos del año 1992 (Ledesma y Zurita, 1995). Se debe considerar que este fue el primer año de evaluación del contenido de Carbono en este pastizal, y que anteriormente en este lugar existían árboles, motivo por el cual el contenido de Carbono en suelo a 0,6 y $1 \mathrm{~m}$ poseen mayores valores acumulados a diferencia de lo registrado en esta serie de suelo, donde no contempla la época en que se realizaron las mediciones (1992) ni hubiesen registros de existencia de árboles en el lugar. Tal como lo manifiesta Mannetje et al. (2008), la diferencia de la cantidad de Carbono a los 0,1 y $0,2 \mathrm{~m}$ de suelo pueden deberse a pérdidas de MOS por mineralización, o impedimentos en la penetración de raíces, por lo cual habría menor contenido de materia orgánica, por ende menor contenido de Carbono.

El contenido de Carbono en la biomasa aérea del pastizal fue de $5,65 \mathrm{tn} \mathrm{ha}^{-1}$, valor mayor al obtenido por Bernardis et al. (2005) quienes encontraron 4,08 th $\mathrm{MS}$ 
$\mathrm{ha}^{-1} \mathrm{año}^{-1}$ en promedio de biomasa y un contenido de Carbono de 1,84 tn ha ${ }^{-1}$, estos autores realizaron un ensayo en un ecosistema de pastizal de $S$. setosum similar al nuestro, con condiciones de clima y suelo similares, con elevadas precipitaciones e irregular distribución y altas temperaturas, y un suelo de textura arcillosa, bajo contenido de materia orgánica, fertilidad de regular a pobre $\left(25^{\circ} 10^{\prime}-28^{\circ} 00^{\prime}\right.$ lat. Sur, $58^{\circ} 15^{\prime}-59^{\circ} 20^{\prime}$ long. Oeste). Valores de contenido de Carbono menores también fueron hallados por da Silva et al. (2004) en pastura nativa y en Brachiaria decumbens, de 0,615 tn ha $\mathrm{h}^{-1} \mathrm{y}$ de 0,42 tn ha ${ }^{-1}$ respectivamente, bajo pastoreo en la zona de El Cerrado, Brasil. Así mismo, Etchevers et al. (2002) en México, obtuvieron valores de contenidos de Carbono menores en pastizales dedicados al pastoreo, de 2,18 tn ha $^{-1}$. Esto podría deberse, según Da Silva et al. (2004), a que tanto la pastura nativa como la cultivada se encontraban bajo un proceso de degradación y como consecuencia la captura de Carbono fue menor. Según Fisher et al. (1994), la combinación de gramíneas con sistemas radicales más profundos con leguminosas fijadoras de nitrógeno pueden incrementar los nutrientes de la biomasa y del suelo, mejorando de una manera más ecológica y sostenible la producción animal.

\section{CONCLUSIONES}

El stock de Carbono en primavera en el ecosistema de pastizal de Sorghastrum setosum es 139,24 tn $\mathrm{ha}^{-1}$, del cual aproximadamente el 95,94\% corresponde a lo acumulado en suelo y el resto en menor proporción corresponde al Carbono en la biomasa aérea del pastizal.

\section{BIBLIOGRAFÍA}

Álvarez, R.; Steinbach, H. y Grigera, S. 2004. Producción de rastrojos por cultivos de trigo en la Pampa Ondulada. VI Congreso Nacional de Trigo (Argentina), Actas en CD.

Álvarez, R.; Steinbach, H. S.; Lavado, R. S. y Gutiérrez Boem, F. H. 2006. Materia Orgánica. Valor Agronómico y Dinámica en Suelos Pampeanos. Editorial Facultad Agronomía. Universidad de Buenos Aires. $1^{\mathrm{a}}$ ed. $256 \mathrm{p}$.

Bernardis, A.C.; Roig, C. y Bennasar Vilches, M. 2005. Prouctividad y calidad de los pajonales de Sorghastrum setosum (Grises.) Hitchc. en Formosa, Argentina. Agricultura Técnica (Chile). Vol. $65 \mathrm{~N}^{\circ} 2$.

Cabrera, A. 1994. Regiones Fitogeográficas Argentinas. Enciclopedia Argentina de Agricultura y Jardinería. Fascículo I. Tomo I.

Cambardella, C. A. and Elliot, E. T. 1992. Particulate Soil Organic-Matter Changes across a Grassland Cultivation Sequence. Soil Science. Society American Journal. 56: 777-783.
Conant, R.T.; Paustian, K.; Del Grosso, S.J. y Parton, W. J. 2005. Nitrogen pools and fluxes in grassland soils sequestering carbon. Nutrient Cycling in Agroecosystems 71:239-248.

Corazza, E.J.; Da Silva, J.E.; Resck, D.V.S.; Gomes, A.C. 1999. Comportamento de diferentes sistemas de manejo como fonte ou depósito de carbono em relação à vegetação de Cerrado. Rev. Bras. Ciện. Solo 23, 425-432.

Da Silva, J.E.; Resck, D.V.S.; Corazza, E.J. and Vivaldi, L. 2004. Carbon storage in clayey Oxisol cultivated pastures in the "Cerrado" region, Brazil. Agriculture, Ecosystems and Environment 103: 357-363.

Dixon, R.K. 1995. Agroforestry systems: sources or sinks of greenhouse gas? Agroforestry systems 31: 99-116.

Etchevers, J.; Acosta, M.; Monreal, C.; Quednow, K. y Jiménez, L. 2002. Los Stocks de Carbono en Diferentes Compartimientos de la Parte Aérea y Subterránea en Sistemas Forestales y Agrícolas de Ladera en México. World Series Vol. 13. IUFRO Secretariat, Vienna. ISSN 1016-3263 - ISBN 3-901347-31-3

FAO. 2002. Captura de carbono en los suelos para un mejor manejo de la tierra. Organización de las $\mathrm{Na}-$ ciones Unidas para la Agricultura y la Alimentación. ISBN 92-5-304690-2

Fisher, M.J.; Rao, I.M.; Ayarza, M.A.; Lascano, C.E.; Sanz, J.I.; Thomas, R.J. and Vera, R.R. 1994. Carbon storage by introduced deep-rooted grasses in the South American savannas. Nature 371: 236-237.

Houghton, J. T.; Y. Ding; D. J. Griggs; Noguer, M.; Van der Linden, P. J. y X. D. 2001. IPCC Third Assessment Report: Climate Change 2001. The Scientific Basis Contribution of Working Group I to the Third Assessment Report of the Intergovernmental Panel on Climate Change (IPCC). Cambridge University Press, UK.

InfoStat. 2007. Infostat version 1.1. Grupo Infostat, Facultad de Ciencias Agrarias, Universidad Nacional de Córdoba, Argentina.

IPCC. 2000. Land use, land-use change, and forestry special report. Cambridge University Press 377 pp.

IPCC, 2001a: Climate Change 2001: The Scientific Basis. Contribution of Working Group I to the Third Assessment Report of the Intergovernmental Panel on Climate Change [Houghton, J.T., Y. Ding, D.G. Griggs, M. Noguer, P.J. van der Linden, X. Dai, K. Maskell, y C.A. Johnson (eds.)]. Cambridge University Press, Cambridge, Reino Unido y Nueva York, NY, Estados Unidos, 881 págs.

IPCC, 2001b: Climate Change 2001: Impacts, Adaptation, and Vulnerability. Contribution of Working Group II to the Third Assessment Report of the Intergovernmental Panel on Climate Change [McCarthy, J.J., O.F. Canziani, N.A. Leary, D.J. Dokken, y K.S. White (eds.)]. Cambridge University Press, Cam- 
bridge, Reino Unido y Nueva York, NY, Estados Unidos, 1031 págs.

IPCC, 2001c: Climate Change 2001: Mitigation. Contribution of Working Group III to the Third Assessment Report of the Intergovernmental Panel on Climate Change [Metz, B., O.R. Davidson, R. Swart, y J. Pan (eds.)]. Cambridge University Press, Cambridge, Reino Unido y Nueva York, NY, Estados Unidos, 752 págs.

Ledesma L. y Zurita J. 1995. Carta de Suelo de la Estación Experimental Agropecuaria Colonia Benítez. L. Ledesma, J. Zurita. E.E.A. R. Sáenz Peña.
Mannetje, L.'t; Amézquita, M. C. and Buurman P. (eds) 2008. Carbon Sequestration in trocal Grassland Ecosystems. Wageningen Academic Publishers. 224 pp.

Prause, Juan. 2006. Análisis de Suelos. Técnicas de Muestreos de suelos, aguas y plantas. Bases prácticas para la fertilización. Consejo Profesional de Ingenieros Agrónomos del Chaco.

Walkey, A.; and Black, A. 1934. An examination of the Degjareff method for determining soil organic matter and a proposed modification of the chromic acid titration method. Soil Science, 37: 29-38. 